\title{
Síndrome de Capgras: una revisión breve.
}

\author{
Capgras syndrome: a brief review.
}

\author{
Mariella Strobbe-Barbat 1,2,a, Luis Macedo-Orrego ${ }^{1, \mathrm{~b}}$, Lizardo Cruzado ${ }^{1,2, \mathrm{~b}}$. \\ RESUMEN
}

Los síndromes de falsa identificación, grupo de patologías neuropsiquiátricas, con las delusiones de falso reconocimiento como denominador común, incluyen, entre otros, al Síndrome de Capgras, caracterizado por la firme creencia, por parte del paciente, de que una persona cercana a él/ella, generalmente un familiar, ha sido sustituida por un "doble exacto" o "impostor", describiendo la similitud física entre ambos; en pocos casos, tal creencia se extiende a animales domésticos e incluso a objetos inanimados. Un alto porcentaje de casos se asocia a un trastorno orgánico cerebral de base, especialmente demencias neurodegenerativas, pero también puede ocurrir en enfermedades psiquiátricas primarias, tales como esquizofrenia y trastornos esquizoafectivos o afectivos. La exploración neurobiológica plantea diferentes hipótesis explicativas del síndrome, entre ellas los modelos de desconexión visuoanatómico de Ellis y Young, de lateralización de Gainotti y de distribución de redes neuronales de Gobbini y Haxby. El falso reconocimiento puede dar lugar a conductas violentas del paciente contra las personas implicadas como supuestos "dobles", especialmente en casos de pacientes varones, con enfermedad psiquiátrica de fondo (i.e., esquizofrenia paranoide), con antecedentes de consumo de sustancias psicoactivas o presencia concomitante de alucinaciones auditivas imperativas. No existe tratamiento específico para el Síndrome de Capgras, pero ciertamente el cuadro psiquiátrico subyacente debe ser manejado con fármacos antipsicóticos e incluso terapia electroconvulsiva.

PALABRAS CLAVE: Síndrome de Capgras, trastornos psicóticos, reconocimiento facial, trastornos neurocognitivos.

\section{SUMMARY}

The false identification syndromes, a group of neuropsychiatric pathologies with delusions of false recognition as their common denominator, include among others, the Capgras Syndrome, characterized by the patient's firm belief that a person close to him/her, usually a relative, has been replaced by an "exact double" or "impostor", describing the physical similarity between both; in a few cases, such belief encompasses domestic animals or inanimate objects. A large percentage of cases are associated with a basic organic disorder, especially neurodegenerative dementias, but it can also occur in a basic psychiatric illness such as paranoid schizophrenia, schizoaffective or affective disorders. Among the neurobiological explanations of the Capgras Syndrome, different models have been described: visuoanatomic disconnection by Ellis and Young, lateralization by Gainotti, neuronal networks distribution by Gobbini and Haxby. The false recognition can lead to violence against the people involved, especially by male patients with an underlying psychiatric illness (i.e., paranoid schizophrenia), a history of psychoactive substance use or the presence of imperative auditory hallucinations. There is no specific treatment for Capgras Syndrome but, certainly, management of the underlying psychiatric condition with antipsychotic drugs and even electroconvulsive therapy, should be initiated.

KEYWORDS: Capgras syndrome, psychotic disorders, facial recognition, neurocognitive disorders.

\footnotetext{
Instituto Nacional de Salud Mental "Honorio Delgado - Hideyo Noguchi”. Lima, Perú.

Facultad de Medicina Alberto Hurtado, Universidad Peruana Cayetano Heredia. Lima, Perú.

Facultad de Medicina de San Fernando, Universidad Nacional Mayor de San Marcos. Lima, Perú.

Médico residente de Psiquiatría ; ${ }^{b}$ Psiquiatra.
} 


\section{INTRODUCCIÓN}

Los síndromes de falsa identificación abarcan un grupo de patologías neuropsiquiátricas cuyo común denominador son las delusiones de falso reconocimiento, es decir, delusiones acerca de la identidad de otras personas. La identidad puede ser explicada por un rango amplio de diferentes perspectivas, y ser definida de varias formas: como un estado subjetivo de continuidad a lo largo del tiempo, como una estructura nuclear interna de la personalidad madura, como identificación con un grupo social, como el sentido del self en tanto un centro estable de actos y comportamientos, entre otros (1).

Existen cuatro principales variantes del síndrome de falsa identificación delusional: síndrome de Capgras, síndrome de Frégoli, síndrome de dobles subjetivos y síndrome de intermetamorfosis. Aparte de estos cuatro cuadros, hay una miríada de reportes sobre inusuales variantes. Si bien hay diversas propuestas de clasificación, no existe ninguna aceptada por consenso sobre este tópico.

En 1981, Christodoulou y Maliana-Loulaki clasificaron estos síndromes en falso reconocimiento delusional positivo y negativo $(2,3)$. En los síndromes de falso reconocimiento delusional positivo, existe un hiperreconocimiento de las personas (personas extrañas son reconocidas como conocidas, al afirmar similitudes que no existen). En los síndromes de falso reconocimiento delusional negativo, existe un hiporreconocimiento de las personas (un conocido es visto como extraño, y se perciben diferencias inexistentes en esta persona, lo que resulta en que se niega su identidad e incluso se afirma que es un impostor) (4). Además, en 1990, Silva y colaboradores clasificaron estos síndromes según la persona cuya identidad es alterada (sí mismo u otras personas), y si la alteración es de carácter físico y/o psicológico (5) (tabla1).

Existen múltiples críticas hacia las clasificaciones de los síndromes de falsa identificación. Primero, no hay acuerdos acerca de la definición exacta de los vocablos utilizados en las clasificaciones, como "transformación", "impostor", "sustitución", etc, por lo que la misma palabra puede ser interpretada en diferentes significados. Por otro lado, se utilizan los términos ya mencionados para clasificar la enfermedad, pero no siempre se recoge la verdadera experiencia fenomenológica del paciente, que muchas veces puede ser incomprensible para sí mismo (y aún más difícil le resultará ponerle nombre a tal experiencia), utilizando otras palabras como "reencarnación" o "posesión del cuerpo". Por lo tanto, las clasificaciones existentes tienen definiciones imprecisas y terminología difusa (6).

El síndrome de Capgras fue descrito por primera vez en 1923 en Francia por J. Capgras y ReboulLachaux, quienes acuñaron el término l'illusion des sosies (o la ilusión de los dobles). En el síndrome de Capgras, el paciente cree que una persona cercana a él ha sido sustituida por un "doble exacto" o "impostor" (palabras frecuentemente utilizadas por el mismo paciente); la delusión se basa en el hiporreconocimiento de una persona específica (7).

El síndrome de Frégoli, descrito por Courbon y Fail, psiquiatras igualmente franceses en 1927, es un síndrome de hiperidentificación, en el cual el paciente identifica a un presunto perseguidor en cualquier persona de su entorno, extraña o no, pese a que ambos no comparten ninguna semejanza física. El paciente acusa al perseguidor de cambiar constantemente su rostro y apariencia, por lo que este síndrome fue bautizado con el nombre del famoso actor italiano, Leopoldo Fregoli, quien era reconocido por constantemente cambiar de apariencia durante sus presentaciones, y era conocido como "el transformista" $(2,8,9)$.

En el síndrome de intermetamorfosis, descrito luego de pocos años, en 1932 por Courbon y Tusques, el paciente cree que la persona ha sufrido una transformación física y psicológica, convirtiéndose en una persona diferente y viceversa; es decir, el paciente cree que dos personas cercanas a él intercambian sus identidades (10).

Finalmente, en 1978, Christodoulou describió el síndrome de dobles subjetivos, en el cual el paciente cree que otras personas se han transformado físicamente, pero no psicológicamente, en el propio paciente. Esto lleva a que el paciente crea que existe un doble de sí mismo y lo logra identificar, pero que actúa independientemente de él.

Los cuatro síndromes de falsa identificación delusional clásicos, tienen cada uno una variante inversa; en el síndrome de Capgras inverso el paciente cree que él mismo vive como "impostor" en un cuerpo que no es suyo; en el síndrome de Frégoli inverso, el paciente cree que su propia apariencia física sufre cambios radicales; en el síndrome de dobles subjetivos inverso el paciente cree que su propia identidad 
psicológica ha sido alterada, y en el síndrome de metamorfosis inversa el paciente tiene la creencia de que ambas, su apariencia física e identidad psicológica, han sido alteradas $(6,11)$.

El conocimiento de estos síndromes de rara presentación por parte del psiquiatra no solo radica en la riqueza psicopatológica, sino en el manejo de los mismos, debido al alto grado de violencia asociado y la posible patología orgánica subyacente en un porcentaje importante de pacientes que los presentan. Desde un punto de vista académico estos síndromes presentan una oportunidad para el estudio e investigación del origen o correlato biológico de los fenómenos delusivos y las funciones fisiológicas subyacentes al reconocimiento de otros individuos.

\section{Historia}

Joseph Capgras nació en Verdun-sur-Garrone, Francia, en 1873. En 1898 inició la especialidad de psiquiatría en los Asilos del Sena. Posteriormente, ejerció en el Hospital Maison-Blanche, y luego en el hospital de Sainte-Anne, hasta su jubilación. Fue discípulo de Magnan, Alix Joffroy y Paul Sérieux. Junto con Paul Sérieux, escribió en 1909 el libro "Las locuras razonantes. El delirio de interpretación”, obra clave en el estudio histórico de la paranoia (12).

El reporte que le dio el nombre al síndrome de Capgras, escrito por Capgras y Reboul-Lachaux en 1923, describió a una mujer de 53 años, de oficio costurera, con más de quince años de enfermedad, quien creía de manera delusional que ella provenía de la realeza, que era la heredera de una gran fortuna, pero que fue sustituida al nacer. De sus cinco hijos murieron tres, y ella atribuyó dos de esas muertes a un envenenamiento realizado por enemigos, y una de las muertes a un secuestro. Además, refería que su hija había sido cambiada en múltiples ocasiones por otras niñas, relatando que llegó a tener más de 2000 de estas niñas sustitutas en un periodo de 5 años. Creía también que su esposo había sido asesinado, y que había dobles que lo sustituían, habiendo por lo menos 8 dobles diferentes. Al ser internada, creía que prácticamente todo el personal tenía dobles incluyéndose a sí misma, y había también dobles de los dobles. Al parecer también tenía alucinaciones auditivas (13).

Sin embargo, la primera descripción del que luego se llamó síndrome de Capgras fue dada por Kahlbaum en 1866, quien había descrito el caso de una paciente que creía que había sido reemplazada por dobles (14).
Posteriormente, otros autores también reportaron descripciones de casos similares, tales como Magnan (1893) y Janet $(1903)(15,16)$. En 1913, Bessiere publicó el caso de una paciente psiquiátrica, quien creía que había dos impostores, uno con semejanza muy similar a la paciente, y otro con semejanza con su madre, y que se aprovechaban de esto para cometer todo tipo de delitos $(17,18)$. En los Estados Unidos de América, Clifford Beers publicó su autobiografía A mind that found itself en 1910, donde describe las delusiones de falso reconocimiento tipo Capgras que él mismo sufría. Pese a todo, el epónimo de Capgras permanece válido por la caracterización más detallada y relevante del fenómeno que después llevó su nombre.

\section{Características clínicas}

El inicio del síndrome de Capgras se puede dar en cualquier momento de la enfermedad a la que acompaña, predominando el cuadro clínico de este síndrome sobre la enfermedad de base. Existe la delusión de que un impostor ha tomado el lugar de una persona conocida, reconociendo la similitud física entre ambas, aunque recalcando sutiles diferencias entre ambos que permiten al paciente distinguir suspuestamente a uno de otro. La delusión es discriminativa, es decir, involucra personas cercanas, generalmente familiares, aunque puede haber una extensión a personas (no familiares) muy cercanas al paciente (7). Silva, en una serie de 29 casos, describe la frecuencia específica de cada "impostor", siendo mayor la frecuencia en familiares directos ( 8 pacientes falsamente identifican a su madre, 5 a su padre, 4 a un hermano y 6 a su esposa), aunque se encontró también la falsa identificación en personas no familiares (5 conocidos, 2 policías, 1 celebridad y 4 extraños) (19). La delusión usualmente involucra a múltiples impostores, aunque también puede ser limitada a un único impostor. Ocurre frecuentemente asociado a paranoia, desrealización y despersonalización. Puede ser transitoria o persistente (20).

Existen variantes poco frecuentes del síndrome de Capgras, como aquellos casos relacionados a animales domésticos e incluso a objetos inanimados. Por ejemplo, Abed y colaboradores mencionan el caso de una mujer de 55 años que creía que muchos objetos de su hogar, tales como su ropa, la máquina para lavar ropa y un cuadro en su sala, habían sido reemplazados por unos objetos duplicados que eran casi idénticos a los suyos (21). Darby y colaboradores reportan el caso de un varón de 71 años que creía que su gato había sido reemplazado por un gato impostor, pese a 
que reconocía que el impostor se parecía físicamente al suyo (22). Islam y colaboradores reportan el caso de una mujer de 53 años que estaba convencida de que sus perros habían sido reemplazados por perros impostores casi idénticos a los suyos (aunque con un ligero cambio de color, y con manchas en sitios diferentes), y unas pinturas de su casa y unas plantas de su jardín también habían sido reemplazadas, dándose cuenta de estas últimas porque eran más pequeñas y jóvenes que sus plantas originales (23).

\section{Epidemiología}

El síndrome de Capgras ha sido estudiado básicamente a través de series de casos, dada su baja frecuencia. Luego del reporte inicial de Capgras en 1923, todos los casos que se reportaban eran de mujeres, hasta 1936, cuando se reportó el primer caso de un varón. Sin embargo, Merrin y colaboradores en 1976 realizaron una revisión de la literatura inglesa, encontrando un total de 46 casos reportados, de los cuales $41 \%$ eran varones y $59 \%$ mujeres (24). Asimismo, Joseph en 1994 realizó un estudio de 835 pacientes hospitalizados en una institución psiquiátrica, encontrando que el $3,1 \%$ de ellos presentaban síndrome de Capgras. De ellos, el 58\% eran varones y $42 \%$ eran mujeres. Posteriormente, hicieron una extrapolación de estos datos a todo el estado de Boston, encontrando que la prevalencia en la población total sería de $0,27 \%$ (25).

Salvatore y colaboradores realizaron un estudio con 517 pacientes hospitalizados por un primer episodio psicótico de un trastorno psicótico primario o de un trastorno afectivo con síntomas psicóticos y encontraron que la prevalencia del síndrome de Capgras era de $14,1 \%$, y que no había diferencias en la prevalencia según sexo, edad actual o edad de inicio del cuadro psiquiátrico. Además, encontraron que la prevalencia de los pacientes con episodios psicóticos agudos o relativamente breves (trastorno psicótico agudo, trastorno esquizofreniforme, trastorno psicótico no especificado) era 2,3 veces mayor que aquella con los otros diagnósticos. Más aún, encontraron otros tres factores asociados al síndrome de Capgras:

- Percepciones delusionales schneiderianas.

- Alucinaciones olfatorias.

- Alucinaciones somatosensoriales (26).

\section{Etiología}

Actualmente se estima que un gran porcentaje de los casos de síndrome de Capgras se asocian a un trastorno orgánico de base (los porcentajes varían en la literatura de $25-40 \%$ ), de los cuales el $81 \%$ estarían relacionados a demencias neurodegenerativas $(7,27)$. Dentro de las enfermedades psiquiátricas de base, el síndrome de Capgras ocurre hasta en un $15 \%$ de los pacientes con esquizofrenia, pero encontrándose también asociada en menor proporción a trastornos esquizoafectivos y afectivos (1,3-4,1\%) (28).

La tabla 2 muestra las diferentes etiologías psiquiátricas y orgánicas del síndrome de Capgras.

\section{Origen psiquiátrico vs. Origen orgánico}

Existen sutiles pero importantes diferencias entre la forma de presentación clínica del síndrome de Capgras según su etiología de base, sea funcional u orgánica. Por ejemplo, los pacientes con un cuadro psiquiátrico primario presentan la delusión de reconocimiento a más temprana edad, con un inicio más insidioso, mientras que en la etiología orgánica suele aparecer a edades más tardías, a veces en adultos mayores, coincidiendo con el daño neurológico o la enfermedad neurodegenerativa de fondo, por lo que suelen presentarse de manera brusca.

Tabla 1. Clasificación de los síndromes de falsa identificación

Falso reconocimiento delusional positivo

Síndrome de Frégoli: Alteración de la identidad física de otras personas.

Síndrome de dobles subjetivos: Duplicación de la propia persona mediante la alteración de la identidad física de otras personas.

Síndrome de intermetamorfosis: alteración de la identidad física y psicológica de otras personas.

\section{Falso reconocimiento delusional negativo}

Síndrome de Capgras: Alteración de la identidad psicológico de otras personas. 
Tabla 2. Etiologías del síndrome de Capgras (27-34)

\section{Demencias \\ Enfermedad de Alzheimer \\ Demencia por cuerpos de Lewy \\ Enfermedad de Parkinson \\ Demencia vascular \\ Delirium posictal \\ Epilepsia \\ Tratamiento electroconvulsivo}

\author{
Otros trastornos neurológicos \\ Traumatismos encefalocraneanos \\ Infarto cerebral \\ Hemorragia subaracnoidea \\ Esclerosis múltiple \\ Encefalitis viral \\ Migraña \\ Enfermedad de Fahr \\ Tumor pituitario \\ Encefalopatías (hepática, postraumática) \\ Encefalitis límbica secundaria a linfoma difuso \\ de células B grandes.
}

\section{Tóxicos \\ Cocaína \\ Diazepam \\ Litio \\ Morfina \\ Ketamina \\ Cloroquina \\ Cobre \\ Alcohol \\ Disulfiram \\ Heroína}

\section{Metabólicas \\ Hiperglucemia o hipoglucemia \\ Pseudohipoparatiroidismo \\ Diabetes mellitus \\ Déficit de vitamina B12 y ácido fólico \\ Hipertiroidismo e hipotiroidismo \\ Hiponatremia}

\section{Enfermedades psiquiátricas}

Esquizofrenia

Trastorno esquizoafectivo

Trastornos afectivos (depresión psicótica,

Trastorno bipolar)

Trastorno delusional

Psicosis postparto
Cuando hay un trastorno orgánico de fondo, hay un mayor daño orgánico cerebral, lo cual se manifiesta como un mayor deterioro cognitivo concomitante. Además, es más frecuente que se presenten en conjunto con otros síndromes de falsa identificación delusional. Por el contrario, es raro que presenten otros síntomas paranoides asociados al cuadro, aunque pueden aparecer alucinaciones visuales. Por otro lado, en casos psiquiátricos, si hay una esquizofrenia de fondo, el deterioro cognitivo es menor, y usualmente hay otros síntomas psicóticos asociados, en especial de tipo paranoide.

En el paciente con etiología orgánica, la delusión tiende a estar presente en todo momento, pero los pacientes no buscan explicar de manera racional lo sucedido. Por otro lado, la delusión en los pacientes psiquiátricos puede fluctuar según las recaídas de los síntomas psicóticos o situaciones estresantes y generalmente reaccionan con otros componentes afectivos.

Finalmente, debido a que los pacientes con un trastorno psicótico interpretan la delusión como amenazante, ellos albergan mayores sentimientos de hostilidad hacia el impostor, en comparación con los pacientes con trastorno orgánico de fondo, quienes no solo consideran esta delusión como un engaño benigno, sino pueden incluso desarrollar afectos positivos hacia el supuesto impostor. Esta diferencia es muy importante ya que conlleva a que los pacientes de origen psiquiátrico sean más agresivos hacia el "impostor".

Las diferentes características entre el síndrome de Capgras de origen psiquiátrico y orgánico se resumen en la tabla 3.

\section{Neurobiología}

Como se ha mencionado, las teorías sobre la génesis del síndrome de Capgras fueron de índole psicodinámica durante la época de esplendor del psicoanálisis, es decir durante más o menos cincuenta años y fueron mencionadas por el mismo Capgras desde el segundo reporte que efectuó sobre el tema. Se asumía que el síndrome de Capgras funcionaba como un mecanismo de defensa para encubrir nada menos que los deseos incestuosos del paciente hacia su propio padre o madre. Dado que dichos deseos son reprobables e inconcebibles por el superyó, y generaban odio y culpa, la aparente solución a semejante conflicto era la "separación" del objeto de dichos deseos y la 
Tabla 3. Diferencias en la presentación clínica del síndrome de Capgras según su trastorno de base (28).

\begin{tabular}{lcc}
\hline & Trastorno psiquiátrico & Trastorno Orgánico \\
\hline Inicio & Insidioso & Brusco \\
Grupo etario & Jóvenes & $\begin{array}{c}\text { Adultos mayores } \\
\text { Menos hostilidad, más } \\
\text { adaptabilidad }\end{array}$ \\
Relación hacia el "impostor" & Hostil & Raros \\
Síntomas paranoides & Usualmente presentes & Mayor \\
Deterioro cognitivo & Menor & Menor \\
$\begin{array}{l}\text { Elaboración de la delusión } \\
\text { Comorbilidad con otros síndromes } \\
\text { de falsa identificación delusional }\end{array}$ & Mayor & Mayor \\
\hline
\end{tabular}

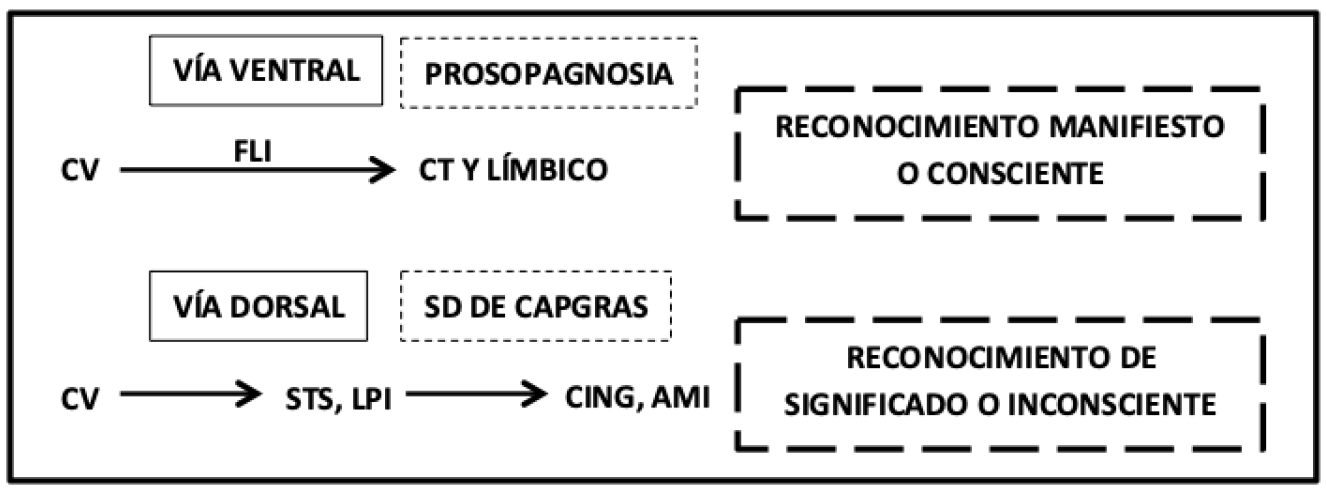

CV: Corteza visual, FLI: Fascículo longitudinal inferior, STS: Surco temporal superior, LPI: Lóbulo parietal inferior, CING: Giro cingulado, AMI: Amígdala

Gráfico 1. Modelo de desconexión visuoanatómico de Ellis y Young adaptado de Bauer (37)

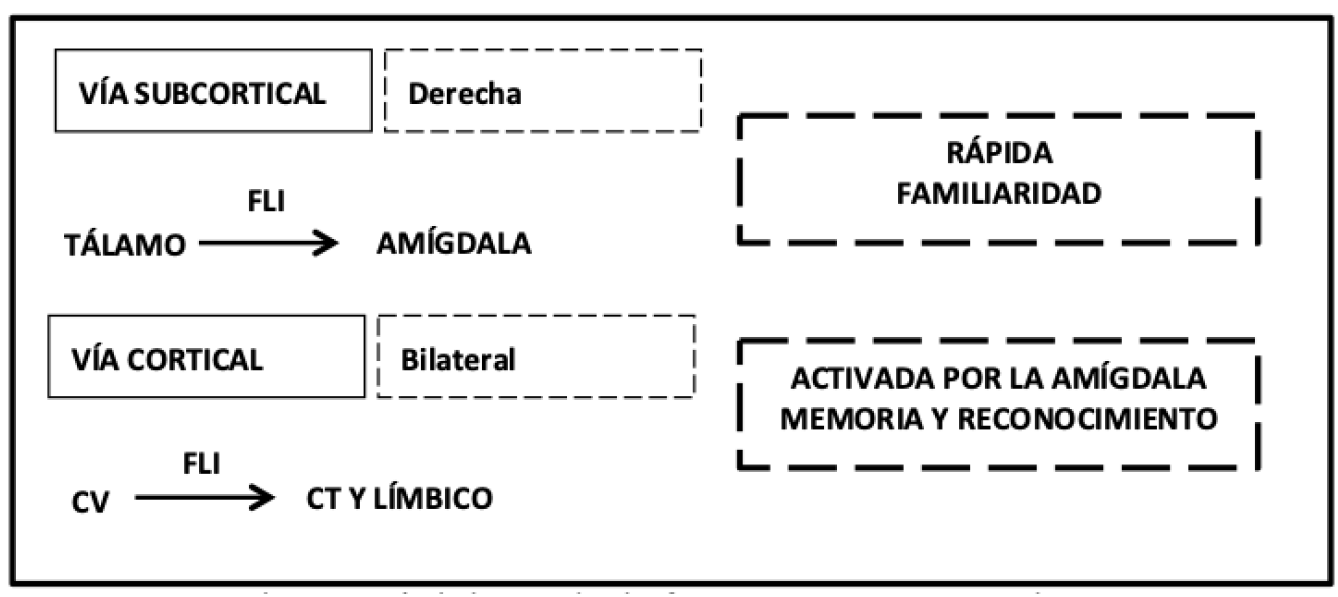

CV: Corteza visual, FLI: Fascículo longitudinal inferior, CT: Corteza temporal.

Gráfico 2. Modelo de lateralización de Gainotti adaptado a partir de Madoz-Gúrpide (38) 
proyección de los sentimientos negativos como el odio, hacia el "impostor". Por supuesto dichos postulados eran insostenibles ante el desarrollo de fenómenos de Capgras ante animales y objetos y con el declive del psicoanálisis han totalmente periclitado. Actualmente, hablar de teorías etiológicas del síndrome de Capgras inevitablemente implica hablar de neurobiología.

En el centro de los fenómenos asociados al Capgras, uno de los primeros que llamó la atención fue el reconocimiento facial, que se estudió desde un punto de vista neuropsiquiátrico, inicialmente en pacientes que presentaban prosopagnosia, es decir, individuos que han perdido la capacidad de reconocer rostros tras una lesión neurológica usualmente de localización inferotemporal derecha (35), estas observaciones han llevado a autores como Atta y cols. a concluir que se debe de considerar la evaluación por neuroimágenes en los casos que son refractarios al tratamiento para descartar lesiones potencialmente tratables (36).

Bauer, en la década de 1980 hizo la observación de que algunos pacientes con este cuadro presentaban signos encubiertos o inconscientes de reconocimiento facial midiendo la respuesta autonómica de conducción de la piel (RACP). La RACP mide indirectamente la resistencia de la piel (la conductividad de la piel es la inversa de la resistencia) y con ello la respuesta autonómica, la cual estaría en relación a la cantidad de sudor en las glándulas ecrinas. Se ha establecido que se presenta un pico en la conductividad de la piel entre 1 a 5 segundos después de presentarse un rostro y que dicho pico es de una mayor amplitud cuando el rostro es conocido (35).

El hecho de que incluso los pacientes con prosopagnosia presenten cambios en la RACP llevó a Bauer a plantear la existencia de dos vías separadas de reconocimiento facial. La primera sería la vía ventral, constituida por el fascículo longitudinal inferior que conecta la corteza visual con el sistema límbico y la corteza temporal, encargada del reconocimiento manifiesto o consciente; y la vía dorsal, que conecta la corteza visual con el surco temporal superior y el lóbulo parietal inferior para finalmente conectarse con la corteza cingulada y la amígdala, la cual a su vez está encargada del reconocimiento inconsciente o de significado. Ellis y Young plantearon un paralelo entre la prosopagnosia y el síndrome de Capgras y plantearon el modelo de desconexión visuoanatómico a partir de la propuesta de Bauer (gráfico 1). De acuerdo a este modelo, en el síndrome de Capgras se vería comprometida la vía dorsal del reconocimiento facial, produciéndose el reconocimiento manifiesto del rostro pero sin la consiguiente adjudicación de significado y contenido afectivo (37).

La principal desventaja de los modelos de desconexión como el de Ellis y Young es que solo explican el síndrome de Capgras visual y no el de Frégoli o de sobreidentificación, además de no explicar el reconocimiento o error de reconocimiento auditivo (28).

Una propuesta más reciente en respuesta a las limitaciones del modelo de desconexión es la del modelo de lateralización de Gainotti (citado por Madoz-Gúrpide) (11), basándose en la observación de que las lesiones izquierdas no afectarían la sensación de familiaridad. En este modelo (gráfico 2) se plantea el reconocimiento facial en dos pasos. Inicialmente se activaría una vía rápida entre el tálamo y la amígdala a la cual el autor denomina vía subcortical, la cual tendría por característica principal estar altamente lateralizada hacia el hemisferio derecho. Posteriormente, la vía subcortical activaría la denominada vía cortical, más lenta, y que sería equivalente a la vía ventral planteada en el modelo de desconexión de Ellis y Young (28).

Finalmente, Gobbini y Haxby plantean el modelo de distribución de redes neuronales. En este modelo los autores hacen énfasis en los tres componentes del reconocimiento de una persona conocida, que son (a) el reconocimiento de la apariencia física, (b) la activación de conocimientos respecto de la persona reconocida y (c) la respuesta emocional asociada, y los agrupan en dos subsistemas. El primer subsistema al que denominan sistema nuclear es el encargado del reconocimiento de la apariencia física. Este primer subsistema estaría compuesto por la corteza temporal superior posterior (encargada del reconocimiento de las características dinámicas), el giro occipital inferior y el giro fusiforme (encargados del reconocimiento de las características invariables). El segundo subsistema o sistema extendido, que ejerce una retroalimentación moduladora descendente y que comprende los conocimientos de la persona y la respuesta emocional. Las estructuras encargadas de la respuesta emocional de este sistema serían la amígdala, ínsula y precúneo, mientras que las relacionadas al conocimiento de la persona serían el giro paracingulado anterior (encargado de reconocer las características personales, actitudes y estados mentales), corteza temporal superior posterior (reconoce las intenciones y estados mentales), corteza temporal anterior (conocimiento biográfico) y la corteza precúneo-giro cingulado 
posterior (memoria episódica). En la disertación de su modelo los autores sostienen que la falla de una respuesta emocional adecuada estaría asociada a la incapacidad de reconocer un rostro como familiar. Es decir, en el proceso de reconocimiento facial se producen los tres procesos anteriormente descritos, el reconocimiento de la apariencia física, la evocación de memoria semántica respecto del rostro reconocido y la respuesta emocional asociada a dicho rostro, y la falla en cualquiera de los tres procesos conllevaría a la imposibilidad de reconocer un individuo como familiar o conocido. En lo correspondiente a la delusión de Capgras no se produciría la respuesta emocional asociada con el rostro reconocido y con ello el individuo lo interpretaría como extraño, pero al producirse los otros dos procesos de forma adecuada el individuo podría interpretarlo como un impostor (38).

Desde luego, aún quedan hilos sueltos en estas teorías neurobiológicas explicativas, por ejemplo, la existencia de otras patologías psiquiátricas con alteraciones en la respuesta galvánica de la piel pero que no acarrean desarrollo de síndrome de Capgras. O también, la interesante presencia de síndrome de Capgras en individuos ciegos, es decir, sin implicación del reconocimiento visual.

\section{Violencia}

En algunos casos, este falso reconocimiento puede llevar a violencia en contra de las personas involucradas, incluso llegar a homicidio. En la literatura, se han encontrado 6 casos detallados de homicidios secundaria a esta patología:

1. Varón de 30 años, con diagnóstico de esquizofrenia paranoide, quien asesinó a su esposa debido a que creía que era un doble y que su verdadera esposa estaba viva, en otro lugar (39).

2. Varón de 37 años con diagnóstico de esquizofrenia paranoide, que creía que sus familiares estaban muertos y habían sido reemplazados por espíritus; mató a su padre debido a que las alucinaciones auditivas que presentaba le decían que el espíritu que controlaba el cuerpo de su padre había anteriormente matado a su tío (quién se había suicidado varios años antes) (40).

3. Mujer de 18 años, con diagnóstico de psicosis secundaria a sustancias psicoactivas, quien creía que sus familiares habían sido reemplazados por impostores. Como no podía encontrar por sus propios medios a su familia, pensó que si era arrestada por un crimen la policía le ayudaría a encontrarlos, por lo que acuchilló a una mujer (41).

4. Mujer de 47 años, con diagnóstico de esquizofrenia paranoide, tenía multiples delusiones de Capgras referentes a varios familiares, y creía que la Sra. $\mathrm{V}$, otra paciente del centro psiquiátrico donde estaba internada, había matado a su hija 16 años atrás. La paciente afirmaba que ahora también esa señora V. quería matar al doble de su hija y concluyó que la única manera de salvar a esa hija era matando a la Sra V, por lo que la acuchilló (42).

5. Varón de 31 años, con diagnóstico de esquizofrenia paranoide, creía que, desde su nacimiento, su madre había sido reemplazada por un francés. El paciente concluyó que la única manera de salvar a sí mismo y al mundo era matando a este francés, por lo que mató a su madre (43).

6. Varón de 21 años, con diagnóstico de psicosis sin etiología identificable, creía que el diablo había reemplazado a su madre. Por lo tanto, asesinó a su madre para salvarse a él, a su hermano y al mundo (44).

Además, si bien no detallan los casos, Nielssen y colaboradores reportaron dos casos de infanticidio secundario a síndrome de Capgras (creían que el niño estaba muerto y había sido reemplazado por un impostor) (45).

Los pacientes más susceptibles a agredir a otros presentan las siguientes características $(11,19,45)$ :

- Enfermedad psiquiátrica de base.

- De presentar esquizofrenia, ser del subtipo paranoide.

- Episodio psicótico activo.

- Pacientes en cuya enfermedad de base se encuentra suspicacia, hostilidad, paranoia.

- Antecedente personal de comportamiento violento (independiente del síndrome de falso reconocimiento delusional).

- Género masculino.

- Antecedente de consumo de drogas.

- Vivir con el "impostor".

- Alucinaciones auditivas imperativas (que ordenan los actos violentos).

- El "impostor" es una persona (en lugar de un objeto).

La violencia, en el caso de una esquizofrenia paranoide o un trastorno delusional, está bien organizada y planificada, usualmente es hacia una persona específica, significante en la vida del paciente 
("el impostor"). Por otro lado, la violencia que ocurre en pacientes con trastornos afectivos, demencia, o esquizofrenia desorganizada, es menos específica y usualmente menos peligrosa (45).

\section{Tratamiento}

No hay un tratamiento específico para el síndrome de Capgras. Debido al gran porcentaje de pacientes con síndrome de Capgras cuya etiología primaria es orgánica, hay que realizar un estudio completo del paciente. Estudios de neuroimágenes y electroencefalograma permitirían buscar causas neurológicas. Para detectar una enfermedad metabólica de base, habría que realizar las pruebas sanguíneas correspondientes para las enfermedades más frecuentemente asociadas a este síndrome (glucosa, hormonas tiroideas, hormona paratiroidea, dosaje de vitamina B12 y ácido fólico). Finalmente, es importante indagar sobre el uso de medicamentos o abuso de sustancias (27).

Posteriormente, se debe de tratar cualquier cuadro psiquiátrico subyacente (orgánico o funcional) e iniciar tratamiento sintomático con fármacos antipsicóticos, $\mathrm{y}$ de ser necesario, terapia electroconvulsiva en aquellos casos resistentes a tratamiento antipsicótico (46). De acuerdo con la etiología, los pacientes pueden también beneficiarse de tratamiento farmacológico con antidepresivos o estabilizadores del ánimo (36). Cabe resaltar que, debido a que la relación del paciente con el "impostor" se deteriora, se debe incluir apoyo a la persona implicada, para que pueda adquirir conciencia de la enfermedad del paciente, y si es necesario, modificar sus actitudes hacia él $(2,7,31)$.

\section{CONCLUSIONES}

El síndrome de Capgras es caracterizado por la creencia de que un impostor de idéntica apariencia ha ocupado el lugar un conocido. Se encuentra asociado a patología orgánica subyacente en aproximadamente el $40 \%$ de los casos, principalmente a cuadros demenciales neurodegenerativos, por lo que se recomienda la evaluación exhaustiva de posibles etiologías orgánicas ante la presencia de este síndrome, especialmente cuando se presenta en adultos mayores. El estudio del síndrome de Capgras ha servido como modelo para el estudio de los circuitos neuronales encargados de la identificación de rostros conocidos. El síndrome de Capgras, así como el resto de los síndromes de falsa identificación delusional, se puede encontrar asociado a conducta violenta por parte de quien lo padece, por lo que debe tomarse en cuenta este aspecto durante la evaluación y manejo de este grupo de pacientes.

A lo largo de su historia como ente conceptual, el síndrome de Capgras ha sufrido los vaivenes de la misma historia de la psiquiatría en el siglo XX. De un enfoque excluyentemente psicoanalítico al principio, donde todo era explicado solo desde los rígidos postulados psicodinámicos, en los últimos 30 a 40 años el síndrome de Capgras se ha "neurobiologizado" y es insoslayable su consideración y análisis desde la perspectiva neuropsiquiátrica más firme (47). Ello no obsta para que el abordaje clínico sea individualizado ni se soslayen los factores psicológicos reactivos al desarrollo del síndrome de Capgras.

\section{Correspondencia:}

Mariella Strobbe-Barbat

Instituto Nacional de Salud Mental "Honorio Delgado - Hideyo Noguchi".

Jr. Eloy Espinoza 709. Urb. Palao. San Martín de Porres. Lima 15102.

Lima, Perú.

Teléfono: (511) 6149200

Correo electrónico: mariella_strobbe@hotmail.com

Declaración de conflictos de interés: Ninguno.

Financiamiento: Autofinanciado.

\section{REFERENCIAS BIBLIOGRÁFICAS}

1. Jorgensen C. Invited essay: identity and borderline personality disorder. J Pers Disord. 2010;24(3):34464. doi: 10.1521/pedi.2010.24.3.344

2. Mesa T. Síndrome de Frégoli: a propósito de un caso. ALCMEON. 2008; 14(4): 24-36.

3. Christodoulou G, Malliara-Loulakaki S. Delusional misidentification syndromes and cerebral “dysrhythmia". Psychiatria clin. 1981;14:245-251.

4. Cavanagh P. Delusional misidentification secondary to perceptual abnormality: An unusual case of Capgras syndrome. Int J Psychiatry Clin Pract. 2000;4(3):2457. doi: $10.1080 / 13651500050518163$

5. Serrano M. Delirios de identificación. Síndrome de Capgras: a propósito de un caso. Revista Gallega de Psiquiatría y Neurociencias. 2002; 1(5): 70-78.

6. Rodrigues A, Banzato C. Delusional misidentification syndrome: why such nosologic challenge remains intractable. Psychopathology. 2006; 39: 296-302.

7. Enoch MD, Ball HN. Síndromes raros en psicopatología. Madrid: Triacastela; 2007.p. 15-36.

8. Chávez H, Warthon D, Demarini M. El síndrome de Capgras: revisión general a propósito de tres casos 
clínicos. Anales de Salud Mental. 1989; 5: 93-101.

9. Courbon P, Fail G. Syndrome d' 'illusion de Frégoli et schizophrénie. Bulletin de la société Clinique de médecine mentale. 1927;20:121-125.

10. Silva J, Leong G, Shaner A, Chang, C. Syndrome of Intermetamorphosis: a new perspective. Comprehensive Phsychiatry. 1989; 30(3): 209-213.

11. Bourget D, Whitehurst L. Capgras Syndrome: A review of the neuropshysiological correlates and presenting clinical features in cases involving physical violence. Can J Psychiatry. 2004; 49(11): 719-725.

12. Álvarez J, Colina F, Esteban R. A propósito de las locuras razonantes del delirio de interpretación (1909) de Paul Serieux y Joseph Capgras. Frenia. 2009;9:135140.

13. Capgras J, Reboul-Lachauz, J. L'Illusion des "sosies" dans un délire systematize chronique. Bulletin de la Societe Clinique de Medecine Mentale. 1923; 11:119133.

14. Mercurio E. Síndrome de Capgras asociado al consumo de sustancias psicoactivas. Revista de Psiquiatría y Salud Mental. 2011;4(2):96-100.

15. Magnan V. Les centres nerveux: Alcoolisme, folies des héréditaires dégénérés, paralysie génerale, médecine légale. Paris: g: masson; 1893. (Fecha de acceso 23 de marzo de 2019) Disponible en: https:// gallica.bnf.fr/ark:/12148/bpt6k769110.texteImage

16. Janet P. Les obsessions et la psychasthénie. Paris: Felix Alcan; 1903. (Fecha de acceso 23 de marzo de 2019) Disponible en: https://archive.org/details/ lesobsessionset 01 janegoog/page/n8

17. Bessiere R. Paranoia et psychose périodique, essai historique clinique, nosographique médico-légal. Tesis. Paris, Francia: Facultad de Medicina de Paris; 1913.

18. Todd J, Dewhurst K, Wallis G. The syndrome of Capgras. Birt. J. Psychiatr. 1981;139: 319-327.

19. Silva J, Leong G, Wernstock R. The dangerousness of persons with misidentification syndromes. Bull Am Acad Psychiatry Law. 1992; 20 (1): 77-86.

20. Cipriani G, Vedovello M, Ulivi M, et al. delusional Misidentification syndromes and dementia: a border zone between neurology and psychiatry. American Journal of Alzheimer's Disease and other dementias. 2013; 28(13): 671-678.

21. Abed R, Fewtrell W. Delusional misidentification of familiar inanimate objects. A rare variant of Capgras Syndrome. British Journal of Psychiatry. 1990; 157(6): 915-917.

22. Darby R, Caplan D. "Cat-gras" delusion: a unique misidentification syndrome and a novel explanation. Neurocase. 2016; 22(2): 251-256.

23. Islam L, Placentini S, Soliveri P, Scarone S, Gambini O. Capgras delusion for animals and inanimate objects in Parkinson's Disease: a case report. BMC
Psychiatry. 2015;15(73):1-5.

24. Merrin E, Silberfarb P. The Capgras Phenomenon. Arch Gen Psychiatry. 1976;33:965-68.

25. Joseph A. Observations on the epidemiology of the delusional misidentification syndromes in the Boston Metropolitan Area: April 1983-June 1984. Psychopathology. 1994;27:150-153.

26. Salvatore P, Bhuvaneswar C, Tohen M, Khalsa H, Maggini C, Baldessarini R. Capgras syndrome in first-episode psychotic disorders. Psychopathology. 2014;47(4):261-269.

27. Bushra I, Ramneesh B, Aniket T, et al. What do we know about delusional misidentificación disorders? A focus on Capgras Syndrome. Neuropsychiatry. 2012; 2(2): 111-116.

28. Madoz-Gúrpide A, Hillers-Rodríguez R. Delirio de Capgras: una revisión de las teorías etiológicas. Rev Neurol. 2010; 50 (7): 420-430.

29. Montesinos J, Salas J, Sánchez L, et al. Síndromes de falsa identificación delirante. Síndrome de Capgras. Presentación de un caso. ALCMEON. 2008; 15(1): 24-32.

30. Salviati M, Saverio F, Macri F, et al. Capgras-like syndrome in a patient with an acute urinary tract infection. Neuropsychiatric Disease and Treatment. 2013; 9: 139-142.

31. Rapinesi C, Kotzalidis G, Del Casale A, et al. Treatment-resistant, five year long postpartumonsetcapgras episoderesolving after electroconvulsive therapy. Int J Psychiatry Med. 2015; 49(3): 227-234.

32. Soares H, Palmeira W, Martins S, Smid J, Nitrini R. Capgras syndrome associated with limbic encephalitis in a patient with diffuse large B-cell lymphoma. Dement Neurophsychol. 2016;10(1):63-69.

33. Lovrecic M, Lovrecic B. Capgras Syndrome in a heroin addict: A case study. Heroin Addict Relat Clin Probl. 2018; 20(1): 7-12

34. Sharma D, Sharma A, Kumar R, Sharma S. Capgras syndrome, diarrhea and hyponatremia. Ann Indian Psychiatry. 2018;2:150-1.

35. Ellis H, Lewis M. Capgras delusion: a window on face recognition. TREND in Cognitive Sciences. 2001; 5(4):149-156.

36. Atta K, Forlenza N, Gujski M, Hasmi S, Isaac G. Delusional misidentification syndromes: Separate disorders or unusual presentations of existing DSMIV categories? Psychiatry. 2006;3(9): 56-61.

37. Ellis H, Young A. Accounting for delusional misidentifications. BJP. 1990; 157:239-248.

38. Gobbini M, Haxby J. Neural systems for recognition of familiar faces. Neuropsychologia. 2007;45:32-41

39. Casu G, Cascella N, Maggini C. Homicide in Capgras' Syndrome. Psychopathology. 1994; 27: 281-284.

40. Silva J, Leong G, Weinstock R, Boyer C. Capgras Syndrome and Dangerousness. Bull Am Acad Psychiatry Law. 1989; 17(1): 5-14. 
41. Kimura S, Inamoto $Y$, Katsurada T. A rare case of Capgras syndrome observed in wake-amine induced psychosis. Folia Psychiatr Neurol Jpn. 1981; 35(1):43-54.

42. O'Reilly R, Malhotra L. Capgras syndrome: an unusual case and discussion of psychodynamic factors. Brit. J. Psychiatr. 1987;151:263-265.

43. Caraballese F, Rocca G, Candelli C, Catanesi R. Mental illness, violence and delusional misidentifications: the role of capgras' syndrome in matricide. J Forensic Leg Med. 2014; 21:9-13. doi: 10.1016/j.jflm.2013.10.012

44. Nielssen O, Large M, Westmore B, Lacjersteen S. Chile homicide in New South Wales from 1991 to 2005. MJA. 2009;190(1):7-11.
45. De Pauw K, Szulecka T. Dangerous delusions. Violence and the misidentification syndromes. Br J Psychiatry. 1998; 152 (1): 91-96.

46. Junho B, Pena R, Vinicius J, Mantovani L. Capgras síndrome in a first-episode, late-onset and superrefractory schizophrenia case. Arch Clin Psychiatry. 2018;45(3):77.

47. Draaisma D. Echos, doubles, and delusions: Capgras Syndrome in science and literature. Style. 2009; 43: 429-441.

Recibido: 22/02/2019

Aceptado: 25/03/2019 